\title{
Technè
}

La science au service de l'histoire de l'art et de la préservation des biens culturels

$40 \mid 2014$

Thérapéia. Polychromie et restauration de la sculpture dans l'Antiquité

\section{La thérapéia des sculptures en Grèce ancienne : le témoignage des sources textuelles}

The therapeia of sculptures in Ancient Greece : evidence provided by textual sources

\section{Evridiki Leka}

\section{OpenEdition}

\section{Journals}

Édition électronique

URL : https://journals.openedition.org/techne/3542

DOI : 10.4000/techne.3542

ISSN : 2534-5168

\section{Éditeur}

C2RMF

\section{Édition imprimée}

Date de publication : 26 novembre 2014

Pagination : 60-68

ISBN : 978-2-7118-6218-4

ISSN : $1254-7867$

\section{Référence électronique}

Evridiki Leka, «La thérapéia des sculptures en Grèce ancienne : le témoignage des sources textuelles », Technè [En ligne], 40 | 2014, mis en ligne le 24 juillet 2020, consulté le 09 février 2022. URL : http:// journals.openedition.org/techne/3542 ; DOI : https://doi.org/10.4000/techne.3542

\section{(c) $(1)$}

La revue Technè. La science au service de l'histoire de l'art et de la préservation des biens culturels est mise à disposition selon les termes de la Licence Creative Commons Attribution - Pas d'Utilisation Commerciale - Pas de Modification 4.0 International. 


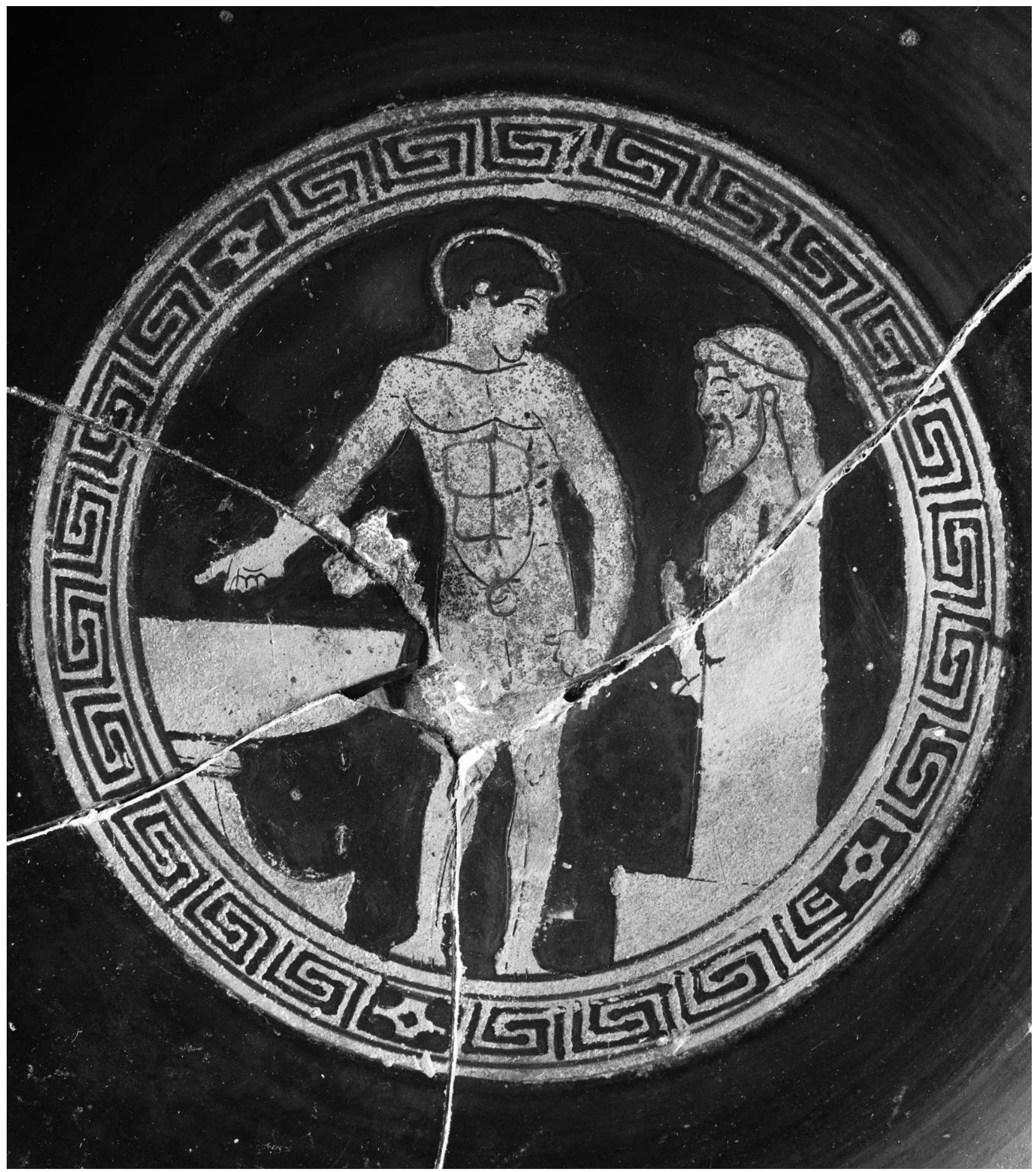

60

Fig. 1. Coupe à figures rouges de type B, inv. Cp 10994, Étrurie, $2^{\mathrm{e}}$ quart du $\mathrm{V}^{\mathrm{e}}$ siècle av. J.-C. Dans le médaillon : un éphèbe nu s'apprête à puiser dans un loutérion pour laver un hermès. @ 1992 Musée du Louvre/Patrick Lebaube. 
Evridiki Leka

\section{La thérapéia des sculptures en Grèce ancienne: le témoignage des sources textuelles}

The therapeia of sculptures in Ancient Greece: evidence provided by textual sources

\begin{abstract}
Résumé. L'étude des textes littéraires et épigraphiques constitue le point de départ indispensable de l'enquête sur la restauration d'œuvres sculptées dans l'Antiquité. Quoique nous ne connaissions à ce jour aucun traité antique sur la conservation et la restauration en général, et encore moins concernant les sculptures, le bilan des informations, forcément ponctuelles et dispersées, voire parfois difficiles à interpréter, qui sont contenues dans les sources textuelles, nous conduit à penser que les Anciens se souciaient beaucoup d'entretenir et de restaurer les cuvres sculptées. Le caractère de leur démarche était cependant très différent du nôtre, comme l'attestent à la fois la nomenclature et les types des traitements, la nature des æuvres traitées, les circonstances et les lieux où les opérations avaient lieu, ainsi que l'identité des personnes qui en étaient responsables.

Mots-clés. Thérapéia, ganôsis, kosmésis, épikosmésis, épiskevè, Olympie, Délos, Ôphélion, Télésinos, Aristandros, Damophon
\end{abstract}

\begin{abstract}
The study of literary and epigraphic texts is the indispensable starting point for the research into the restoration of sculptures in Antiquity. Although no ancient treatise on conservation or restoration in general, and even less of sculpture, is known to exist today, occasional information gleaned here and there from textual sources, despite being sometimes hard to interpret, leads us to think that the Ancients cared a great deal about maintaining and restoring sculptural works. The nature of their approach was, however, very different from ours, as can be seen from the nomenclature and types of treatment, the kind of works treated, the circumstances and places in which these operations occurred and the identity of the people in charge of them.
\end{abstract}

Keywords. Therapeia, ganosis, kosmesis, epikosmesis, episkeve, Olympia, Delos, Ophelion, Telesinos, Aristandros, Damophon.

\section{Introduction}

Les études générales ayant jusqu'ici abordé le sujet de la restauration des sculptures dans l'Antiquité tendent à montrer qu'il s'agissait d'une pratique courante à l'époque hellénistique et romaine, mais négligent en principe les périodes antérieures. Il est d'ailleurs vrai que dans le contexte culturel des royaumes hellénistiques et de l'Empire romain, qui favorisait l'apparition de phénomènes liés à la production artistique, tels les musées et les collections, les copies, le marché et la critique d'art, la restauration des œuvres sculptées apparaît plus ou moins attendue; à Rome, elle était même institutionnalisée. Au contraire, en Grèce archaïque et classique, le climat culturel ne semble pas, du moins jusqu'au IV ${ }^{\mathrm{e}}$ siècle av. J.-C., avoir favorisé l'apparition de tels phénomènes. Toutefois, les études plus spécialisées portant sur les aspects techniques de la sculpture archaïque et classique suggèrent que les restaurations antiques y furent nombreuses et variées. Qui plus est, les sculptures archaïques et classiques qui n'ont pas subi de modifications liées au collectionnisme romain ou moderne permettent une identification plus aisée des interventions antiques. Il faut songer aussi aux effets de la pérennité de ces œuvres à travers le temps : les restaurations effectuées notamment à l'époque hellénistique et romaine ont pu contribuer au contact direct des «sculpteurs-restaurateurs» avec les œuvres vénérables du passé; elles ont pu de ce fait jouer un rôle dans la longévité de certains types statuaires archaïques et classiques, ainsi que dans l'émergence de styles rétrospectifs, comme l'archaïsme et le classicisme.

Ces questions ont été explorées dans le cadre d'une thèse de doctorat dont cet article est issu ${ }^{1}$. La recherche a été conçue comme une première approche synthétique du sujet, visant à confronter aux textes littéraires et épigraphiques les éléments acquis par l'examen direct des sculptures. L'objectif poursuivi est de compléter nos connaissances sur les mesures de protection, les opérations d'entretien, ainsi que les techniques de réparation appliquées aux sculptures en Grèce ancienne, dans le but de mieux comprendre les motifs qui poussaient les Anciens à procéder à des interventions destinées à la sauvegarde de ces œuvres. On propose en effet que les manières dont ils choisissaient de procéder dans ce domaine reflétaient essentiellement les valeurs qu'ils assignaient aux œuvres traitées. Les mots ou les phrases employés dans les textes sont à cet égard révélateurs. 


\section{La ganôsis et la thérapéia}

Une inscription du sanctuaire d'Apollon au Ptoion de Béotie

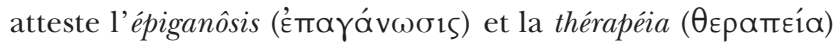
des statues parmi les préparatifs nécessaires à la célébration des jeux et de la grande fête quinquennale des Ptoia, com-

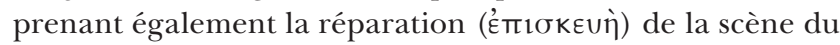
théâtre ${ }^{2}$. Destinées à la remise en état des statues, la ganôsis et la thérapéia étaient confiées à une commission spéciale, à laquelle l'agonothète (le promoteur des jeux) avait remis la somme considérable de 294 drachmes. Apparemment, elles ne concernaient pas seulement la statue cultuelle d'Apollon, mais aussi toutes les statues du sanctuaire qui en avaient besoin, dont un grand nombre de sculptures archaïques, surtout des kouroi, qui ont été découvertes lors des fouilles françaises du site à la fin du XIX ${ }^{\mathrm{e}}$ siècle $^{3}$.

La ganôsis ( $\gamma a ́ v \omega \sigma ı s=$ astiquage) est un terme technique assez spécialisé qui désigne un traitement de surface destiné par excellence à protéger, mais aussi à donner de l'éclat et du brillant au décor polychrome des sculptures ${ }^{4}$. Il s'agissait pour l'essentiel de recouvrir la surface des œuvres d'une légère couche de cire chaude, mélangée à de l'huile, qui, au moyen d'un lustrage soigné, imprégnait le matériau de structure et le revêtement peint ou doré pour les préserver contre l'usure et l'écaillage. L'opération est explicitement décrite par Vitruve, Pline et Plutarque à propos des précautions spéciales prises pour la préservation de la couleur du cinabre ${ }^{5}$. Qui plus est, Plutarque précise que les sculpteurs (oi $\lambda_{1} \theta_{o} \xi$ óol $_{\text {) }}$ avaient recours à la ganôsis pour traiter les parties blessées et

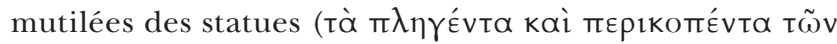

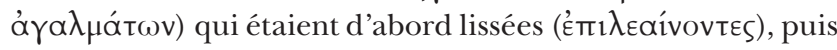

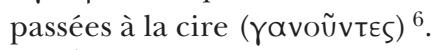

À l'opposé de la ganôsis, la thérapéia est un mot riche en connotations symboliques. Elle désigne concrètement le traitement médical, le service rendu à une personne malade et l'entretien du corps humain, mais aussi, dans un sens plus abstrait, le service rendu à une divinité ou à un personnage important en vue d'obtenir sa faveur. Appliqué à des statues (divines ou autres), ce terme générique résume le contenu et le caractère de la conservation et de la restauration des sculptures en Grèce ancienne, en ce sens qu'il en contient les paramètres essentiels, à savoir la nécessité pratique, les impératifs du culte, l'anthropomorphisme des dieux et de leurs effigies. La thérapéia des statues comprenait toute une série de soins réguliers - lavage, onction avec une huile ou un parfum, habillage, parure, offre de repas rituels -opérés dans les sanctuaires par les prêtres et le personnel auxiliaire au culte afin d'assurer la maintenance des images divines en bon état. En certaines occasions, la thérapéia des statues était confiée à un personnel spécialisé et pouvait désigner des soins plus importants - nettoyages plus poussés, travaux de dorure et de polychromie, réparations ou restitutions de parties mutilées.

La thérapéia des sculptures commençait par le nettoyage. Pausanias (V, 14, 5) note que les phaidryntai ( $\propto \alpha 1 \delta \rho u v \tau \propto i)$, réputés descendants de Phidias, avaient reçu des Eléens le

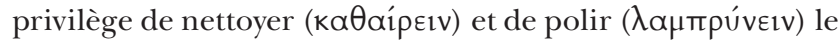
colosse chryséléphantin de Zeus des salissures qui s'y dépo-

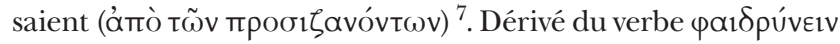

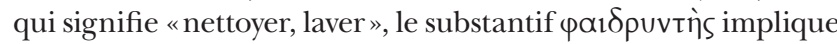
sans doute un nettoyage à l'eau ${ }^{8}$. Pausanias relate aussi qu'avant de commencer à polir la statue, les phaidryntai faisaient des sacrifices à Athéna Erganè, la déesse protectrice des artisans, qui les aidait à mener à bien l'opération. Des inscriptions de l'époque impériale qui ont été trouvées à Olympie et à Athènes honorent des personnes portant le titre de phaidyntai et chargées de nettoyer les statues chryséléphantines de Zeus Olympios ${ }^{9}$. Le fait que le nettoyage des statues était intégré dans le rituel religieux suffit à montrer l'importance de l'enjeu qu'il constituait. Qui plus est, nettoyer des effigies divines particulièrement renommées en raison de leur ancienneté ou de leur finesse technique représentait une tâche honorable, mais aussi très compliquée: pensons aux difficultés énormes impliquées par le nettoyage des colosses chryséléphantins ou par celui d'idoles vêtues et parées de multiples accessoires, nécessitant le lavage des vêtements sacrés et des éléments de la parure. Outre ses fortes connotations symboliques, le nettoyage répondait au besoin précis de tenir propre la statue et ses ex-voto afin de plaire à la divinité et ainsi d'obtenir sa faveur. Il concernait également les sculptures funéraires.

Le nettoyage était complété par divers traitements de surface à base de produits organiques, tels l'huile d'olive

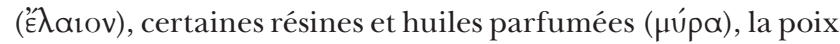
des pins ( textes littéraires et l'épigraphie livrent des informations précises ${ }^{10}$. Appliqués soit par onction, soit par injection, soit par immersion, ces traitements étaient destinés à préserver les matériaux de structure et de revêtement du vieillissement naturel et des altérations, dues aux conditions climatiques et atmosphériques ainsi qu'à l'action nuisible de la végétation et des animaux. Les traitements de surface intervenaient déjà au moment de la finition des œuvres et témoignent avant tout d'une préoccupation de conservation préventive. Une fois les œuvres mises en place, ils étaient répétés à intervalles réguliers dans le cadre de la thérapéia.

L'huile d'olive était amplement utilisée pour la protection de tous les matériaux de la sculpture antique (pierre, bronze, ivoire, bois, etc.). Elle entrait aussi dans la préparation à base de cire d'abeille, appliquée selon l'opération de la ganôsis. Le rôle de l'huile dans la pratique cultuelle conditionne son emploi privilégié dans le traitement des sculptures. Cet usage généralisé tenait aussi à la pureté, aux qualités hydrophobes et curatives, ainsi qu'à l'application aisée d'une substance particulièrement abondante en milieu méditerranéen, qui était de plus vouée aux soins du corps humain. L'onction avec une huile parfumée entrait également dans le traitement du corps humain, un paramètre qui dictait apparemment les choix des Anciens dans le domaine de l'entretien de statues. Mais l'action précise de chaque produit ne leur échappait pas non plus, à savoir par exemple l'action désinfectante des huiles parfumées contre les champignons et les insectes 
xylophages. Pausanias (IX, 41, 7) signale que si les statues en

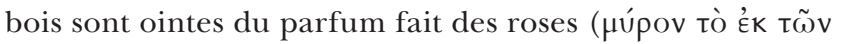

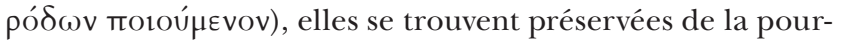

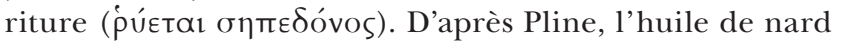
était injectée à travers de nombreux orifices au cœur de la statue d'Artémis à Éphèse (multis foraminibus nardo rigari), afin que le bois conserve son humidité et que les joints ne risquent pas de s'ouvrir (Histoire naturelle, XVI, 214). Les huiles parfumées sont évoquées aussi à propos de la préservation des statues en marbre abritées dans des lieux couverts. La protection des bronzes impliquait également l'emploi de la poix de pin.

Les statues chryséléphantines colossales posaient d'énormes problèmes de conservation, liés à leur technique de fabrication qui associait des matériaux divers: des tôles d'or et des plaques d'ivoire étaient collées sur un noyau en bois soutenu à l'intérieur par un système de poutres de bois disposées en échafaudage ${ }^{11}$. Les auteurs anciens signalent surtout les précautions prises contre la dégradation de l'ivoire, matériau rare, précieux et fragile, qui faisait la gloire de ces statues. Pausanias relate qu'un réservoir peu profond, situé devant la statue de Zeus à Olympie, était destiné à collecter le surplus de l'huile versée

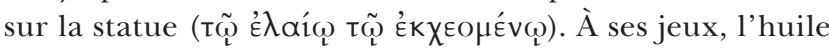
protégeait l'ivoire du dommage causé par l'humidité du

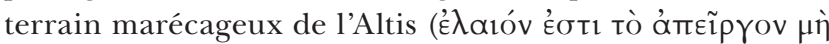

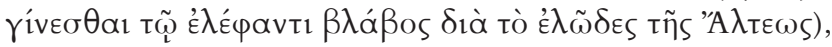
tandis qu'à Athènes, c'était l'eau qui protégeait la statue d'Athéna Parthénos contre la sécheresse de l'Acropole, due

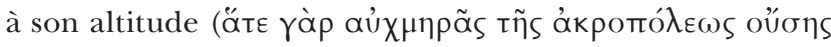

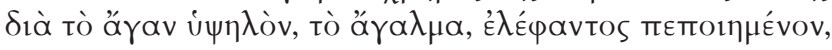

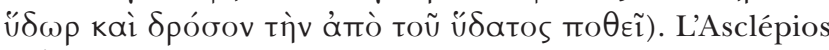
d'Épidaure, quant à lui, était placé au-dessus d'une source

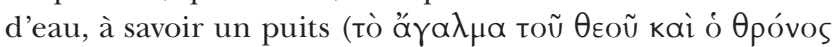

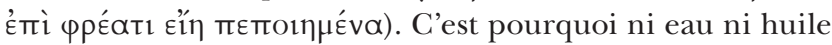

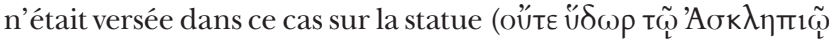

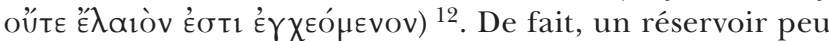
profond a été découvert par la fouille devant les restes du socle de la statue de Zeus, actuellement conservés dans le temple d'Olympie. Quoique le Périégète ne le mentionne pas, un réservoir analogue a été également localisé dans le Parthénon, installé quelque temps après l'aménagement initial du sol du temple ${ }^{13}$. Placés à proximité immédiate des statues, ces réservoirs collectaient les grandes quantités d'huile ou d'eau qui était versées sur les colosses tout en équilibrant l'humidité ou la sécheresse de l'air dans les temples ${ }^{14}$. Le but était de contribuer à ce que l'ivoire retienne dans ses pores le taux d'humidité nécessaire pour qu'il ne se fende pas. Un dispositif très sophistiqué d'approvisionnement en eau a été également découvert dans le temple d'Asclépios à Épidaure, sous le socle de la statue ${ }^{15}$. Méthodios relate que Phidias luimême avait commandé de verser de l'huile devant la statue

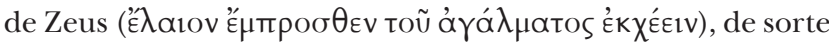
que celle-ci soit conservée immortelle autant que possible (apud Photius, Bibliothèque, 234) ${ }^{16}$. Pline signale que la statue de Saturne à Rome était remplie d'huile (intus oleo repletum est) «un produit considéré comme utile pour protéger l'ivoire de la pourriture» (Histoire naturelle, XV, 32). Il est plus probable qu'à Olympie, l'huile était employée dans un traitement extérieur de l'ivoire, sans doute après le nettoyage à l'eau opéré par les phaidryntai. L'application d'un enduit huileux assez épais visait à bloquer les influences des agents atmosphériques et à maintenir l'humidité intrinsèque de l'ivoire.

\section{La kosmésis}

Mais la thérapéia ne se limitait pas aux sculptures seules. Elle concernait également les vêtements sacrés et les éléments de la parure, à savoir tout ce qui faisait la beauté - le kosmos - d'une statue antique. Les effigies cultuelles en particulier étaient littéralement recouvertes d'accessoires réels qui les paraient, les habillaient et leur donnaient toutes les apparences de la vie: coiffures, perruques, couronnes, vêtements, chaussures, armes, bijoux... Qui plus est, l'habillage des statues faisait partie intégrante du rituel religieux. L'entretien du kosmos impliquait le lavage et le renouvellement régulier des vêtements sacrés, la réparation ou le remplacement des éléments de la parure. Cet aspect de la sculpture antique a forcément été négligé dans les études modernes en raison de la fragilité, voire de la disparition des matériaux, mais son importance se laisse deviner à travers le terme employé dans les textes pour désigner non seulement la remise à neuf du kosmos, mais aussi un ensemble d'opérations d'entretien - nettoyage, traitement de surface, pose ou réfection de peinture et de dorure, réparation d'éléments défectueux, restauration -, le tout étant résumé dans le mot kosmésis. Dérivée du mot

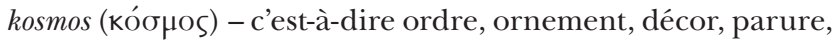

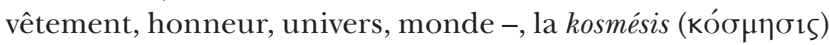
signifie en principe la mise en ordre, l'arrangement, la disposition, mais aussi la décoration et l'embellissement matériel. Appliqué à la sculpture, le mot désigne a) l'habillage et la parure des effigies divines dans le cadre de rites précis, b) la toilette annuelle des statues et la rénovation des sanctuaires lors des préparatifs pour les fêtes et c) la restauration des statues.

C'est grâce aux comptes rendus par les administrateurs des sanctuaires de Délos que l'on arrive pratiquement à reconstituer les opérations comprises dans la kosmésis annuelle des statues et des lieux sacrés ${ }^{17}$. Datant de la fin du IV ${ }^{\mathrm{e}}$ jusque vers le milieu du $\mathrm{II}^{\mathrm{e}}$ siècle av. J.-C., ces textes épigraphiques énumèrent de façon systématique les fournitures acquises, notamment pour la kosmésis de l'Artémision et de l'Héraion

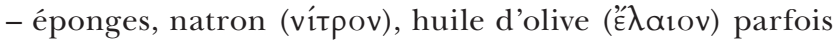

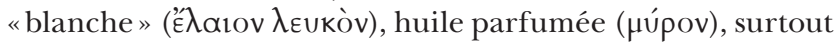

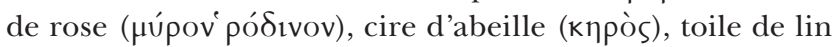
( $\lambda$ ívov), cordon(s) ( $\sigma \varphi \eta ́ \kappa \omega \mu \alpha(\tau \alpha)$ et pinceau de peintre

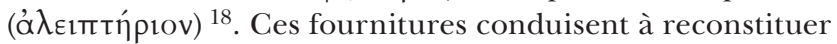
les opérations suivantes: nettoyage à l'eau additionnée d'un produit détergent doux (le natron), onction à l'huile d'olive ou à l'huile parfumée et traitement à la cire de type ganôsis avec emploi de pinceau, de toile de lin et de cordon(s). 
La kosmésis annuelle était destinée à préparer les statues d'Artémis et d'Héra en vue des fêtes consacrées aux déesses. Le nettoyage et la remise en état des temples et des autels entraient également dans ces préparations qui sortaient du cadre des soins quotidiens et incombaient aux prêtres et aux prêtresses ainsi qu'aux fonctionnaires sacrés spécialisés dans la kosmésis, masculins pour Artémis (kosmétai) et féminins pour Héra (kosmousai). Dans les comptes des hiéropes déliens, épikosmésis

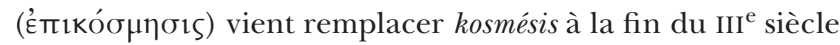
av. J.-C. sans que le préfixe ajouté ne change le sens du terme. La kosmésis annuelle n'était pas réservée aux seules divinités déliennes. À Athènes, les préparatifs pour la fête d'Aphrodite Pandémos comprenaient le lavage des deux statues de culte (celle d'Aphrodite et celle de Peitho), la fourniture d'un nouveau vêtement pour l'effigie d'Aphrodite, la purification du sanctuaire, le blanchiment à la chaux des autels et l'application de poix sur la charpente du temple ${ }^{19}$. Des fonctionnaires sacrés portant les titres kosmétès et kosméteira sont attestés également à Athènes et à Éphèse, tandis qu'à Delphes, ce sont les hiéromnémons qui étaient chargés d'entretenir les sanctuaires, puis de les préparer pour les fêtes panhelléniques ${ }^{20}$.

\section{L'épikosmésis: le cas d'Ôphélion, restaurateur à Délos au III $^{\mathrm{e}}$ siècle av. J.-C.}

Les soins des prêtres et des fonctionnaires sacrés ne suffisaient cependant pas à assurer l'intégrité, la propreté et l'éclat des statues. Lorsque des interventions plus importantes s'avéraient nécessaires, réparations, réfections et remises à neuf étaient confiées aux soins d'artisans spécialisés, voire de sculpteurs renommés. En ce sens, on rencontre surtout les formes verbales du terme épikosmésis et le verbe ou le substantif adjoint fait présumer du type de l'intervention. Dans les comptes de Délos, l'épikosmésis est associée à la peinture à l'encaustique

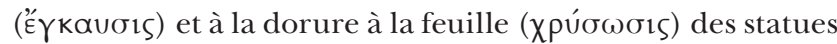
en marbre ou en bois et elle désigne donc des réfections au niveau de la polychromie et de la dorure. Les interventions concernaient aussi bien les statues anciennes que neuves, comme l'agalma de Dionysos en forme d'un oiseau-phallus en bois refait chaque année pour la fête du dieu ${ }^{21}$. La statue en marbre d'Aphrodite, érigée peu avant 304 av. J.-C. dans l'Aphrodision de Stésiléos ${ }^{22}$, fait déjà en 304/3 l'objet d'un traitement de ganôsis confié également à un artisan externe ${ }^{23}$. L'épikosmésis de la même statue en 246 av. J.-C. fait partie des travaux entrepris par Ôphélion (ID 290, 1. 151-153) et implique la réfection de la peinture à l'encaustique et de la dorure avec mention de feuilles d'or ( $\pi \varepsilon ́ \tau \alpha \lambda \alpha)$. À deux reprises supplémentaires, en 224 puis en 218 av. J.-C., l'épikosmésis de la statue d'Aphrodite est spécifiée par l'achat de feuilles d'or, dont le nombre limité suggère des compléments de dorure ${ }^{24}$. Intervenues à intervalles à peu près réguliers, les trois réfections au niveau de la dorure témoignent suffisamment de la fragilité de ce type de décor. D’après les inventaires des fonctionnaires athéniens, établis au $\mathrm{II}^{\mathrm{e}}$ siècle av. J.-C., cette statue tenait de la main droite une phiale en bois doré et portait des boucles d'oreilles en or ${ }^{25}$. Financée par le fonctionnaire

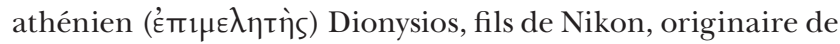

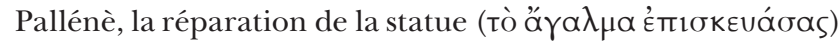
est mentionnée dans deux dédicaces à Aphrodite datant de 110/9 av. J.-C. (ID 1810 et 1811) ${ }^{26}$. Dans l'une, la reconstruction

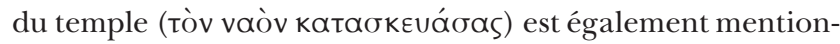
née, tandis que dans l'autre, la réparation concerne aussi les socles (tàsßáoєıৎ) (des statues?). Or, le vieux xoanon archaïque d'Aphrodite conservé au milieu du III $^{\mathrm{e}}$ siècle av. J.-C. dans l'Aphrodision «dans le Sanctuaire» (ID 290, 1. 84) avait, à l'époque de Pausanias, la main droite détériorée par le temps

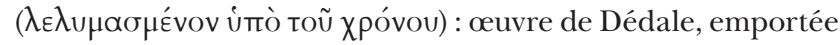
par Ariane depuis la Crète puis consacrée par Thésée à Apollon après sa séparation d'elle, cette statue de petites dimensions se terminait en bas, au lieu de pieds, par un rectangle ${ }^{27}$.

Les passages des comptes des hiéropes portant sur les travaux assignés à Ôphélion au milieu du III ${ }^{\mathrm{e}}$ siècle av. J.-C. nous introduisent dans le contexte de véritables programmes de restauration à grande échelle, à en juger à la fois par les dépenses considérables engagées pour les salaires des artisans et les fournitures, ainsi que par l'organisation des travaux

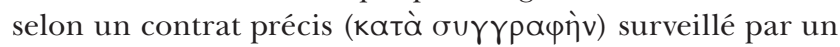
expert (le dénommé Nikénoridès) en accord avec le maître

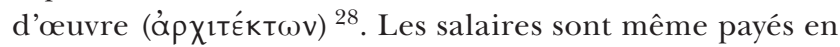
deux versements, une moitié en tant qu'acompte lors de la prise en charge du contrat et le reste à l'achèvement du travail. Qui était Ôphélion? On connaît de ce nom un sculpteur ${ }^{29}$, fils d'Aristonidas ${ }^{30}$, ainsi qu'un peintre ${ }^{31}$, et il est tentant de supposer, d'après les travaux dont il était chargé à Délos, qu'il s'agissait en fait d'une seule et même personne ayant les deux qualités. Toutefois, à en juger par la chronologie d'Aristonidas (vers 340-330 av. J.-C.), la personne qui a travaillé à Délos au milieu du $\mathrm{III}^{\mathrm{e}}$ siècle a peu de chances d'avoir été son fils sculpteur, mais plutôt son arrière-petit-fils, dont la chronologie correspond par ailleurs à celle du peintre Ôphélion. Les formules désignant les tâches dont il a reçu l'adjudication associent à deux reprises l'épikosmésis à la dorure de la statue en marbre d'Artémis-Hécate «sur l'Île» ${ }^{32}$. Dans un cas, l'acquisition de 23 feuilles d'or indique une réfection de dorure assez étendue pour laquelle il a touché 12 drachmes sans compter le prix des feuilles d'or (au total 15 dr. et 1 obole) ${ }^{33}$. Dans l'autre cas, son salaire s'élève à 25 drachmes sans compter le prix de 4 feuilles d'or ( 2 dr.) destinées au carquois de la déesse et qui suggèrent un complément de dorure ${ }^{34}$. La provision de 15 feuilles d'or (coût total $7 \mathrm{dr}$. et 3 oboles) est prévue également dans le contrat qui associe l'épikosmésis à la peinture à l'encaustique de la statue d'Aphrodite de Stésiléos, et attribue 115 drachmes de salaire à Ôphélion ${ }^{35}$. Dans ce cas, l'épikosmésis de la statue est complétée par le blanchiment à la chaux du temple de la déesse, assigné à un autre entrepreneur.

La provision de 1500 feuilles d'or destinées à la dorure des «trois statues du Pythion» et le salaire de 450 dr. attribué à Ôphélion témoignent d'un grand programme de restauration qui comprenait également la peinture à l'encaustique des statues en bois, le raclage de certaines parties qui en 
avaient besoin ( $\xi \tilde{v} \sigma \alpha 1$ tò $\chi \rho \eta і \zeta \zeta o v \tau \alpha)$, ainsi que «la mise en place du reste de la parure comme c'était à l'origine» (kaì

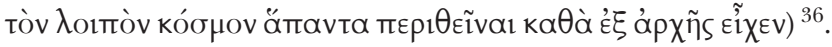
Cette phrase est révélatrice à plus d'un titre: en dehors de la dorure et de la peinture à l'encaustique qui constituaient l'essentiel de la kosmésis, celle-ci renfermait également d'autres éléments, dont il est question plus loin, le tout ayant pour but de remettre les statues dans leur état d'origine. Une lacune dans le texte nous cache toutefois l'identité des parties à racler. Selon la restitution choisie, celles-ci peuvent être soit

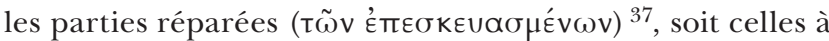
redorer et à repeindre. Le texte mentionne aussi l'achat de

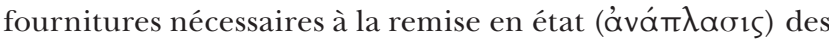

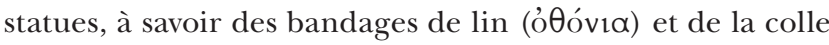
(кó $\lambda \lambda \eta$ ). Les mots anaplasis et othonia appartiennent au lexique de la chirurgie et suggèrent la réparation des parties mutilées, à savoir que les othonia étaient les bandages qui enserraient les pièces rapportées pendant que la colle séchait ${ }^{38}$. La quantité $(5$ mines et demi $=$ env. $2250 \mathrm{gr}){ }^{39}$ et le prix $(30 \mathrm{dr}$. et 4,5 oboles) de la colle achetée sont indicatifs du nombre et de la taille des recollages. Dans ce contexte, le raclage représenterait la finition des parties réparées. Un autre artisan était chargé de réparer les couronnes (toù oteqávous) et le reste de la parure pour la somme de $50 \mathrm{dr}$. La confection

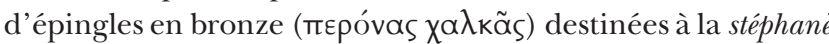
et celle d'éléments non identifiés destinés aux feuilles des

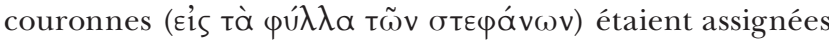
respectivement à Lysimachos et à Eutychos pour 5 et 6 drachmes. Du fait sans doute de leur taille colossale, la restauration des statues du Pythion nécessitait aussi la mise en place d'un échafaudage (tà íkpia), dont le texte mentionne le bois apporté par des ouvriers salariés, la construction, ainsi que les techniciens chargés de cette dernière. Les paiements versés aux entrepreneurs étaient ordonnés par l'expert Nikénoridès, en accord avec le maître d'œuvre et un corps de curateurs

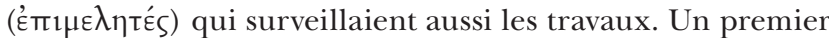
versement est remis à Ôphélion lors de la prise en charge du contrat, puis un deuxième est effectué après l'application des 1500 feuilles d'or et la peinture à l'encaustique des statues. Ôphélion devait recevoir le reste de la somme convenue à l'achèvement des travaux. Or la fin du texte, d'ailleurs mal conservée, laisse supposer qu'un différent a surgi entre l'entrepreneur et les maîtres d'ouvrage : Ôphélion n'aura pas voulu accepter un prix global pour les trois statues, manifestement parce que le travail s'était révélé plus difficile que prévu l'achat d'un supplément de 760 feuilles d'or étant par exemple mentionné dans le texte. Le paiement sera finalement effectué statue par statue ${ }^{40}$.

Le compte des hiéropes déliens de l'an 250 av. J.-C. contient également un paiement de 7 drachmes attribué à deux artisans, Céphalion et Bion, pour avoir opéré l'épikosmésis de «la statue du Dioscure» (tò ơ $\gamma \alpha \lambda \mu \alpha$ toũ $\Delta$ tookoúpou) ${ }^{41}$. Bion, dont le nom revient plusieurs fois dans le même compte ${ }^{42}$, se révèle un artisan spécialisé dans le travail de la pierre. Cette épikosmésis intervient une seule fois à l'avance de l'épikrasis annuelle, un banquet rituel en l'honneur des Dioscures ${ }^{43}$.
Il s'agit sans doute d'une réparation ponctuelle pratiquée sur la statue d'un seul Dioscure plutôt que de la kosmésis annuelle des deux statues avant la fête. La statue cultuelle dont il est question dans ce texte était très probablement un couros archaïque en marbre, réinstallé au début du $\mathrm{III}^{\mathrm{e}}$ siècle av. J.-C., sur une nouvelle base, dans le Dioscourion hellénistique et dont subsistent encore quelques débris, à savoir une partie de la tête, la main droite et un morceau de la plinthe avec l'extrémité antérieure du pied gauche (Musée de Délos, inv. A 3998-4000). Les deux Dioscures - le couros archaïque et son pendant hellénistique - ont été remis en état également au début du I ${ }^{\text {er }}$ siècle av. J.-C., quand le prêtre Athénobios, se vantant d'avoir mis fin à une longue période de disgrâce du sanctuaire, liée sans doute à la domination athénienne de l'île après 169, a «rendu leur splendeur aux idoles des Dioscures, longtemps couvertes par la crasse de l'oubli » (ID 2548) ${ }^{44}$.

Or Pausanias (III, 16, 1) relate qu'à Sparte, au sanctuaire des Leukippides Hilaeira et Phoebe, une prêtresse nommée Leukippis a procédé à l'épikosmésis de l'une des deux statues cultuelles en remplaçant le visage ancien (ávtì toũ ảpxaíou)

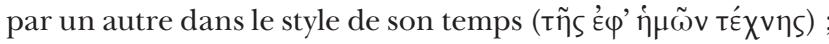
un rêve lui interdit toutefois de restaurer ainsi la deuxième statue $^{45}$. La vue des deux œuvres placées côte à côte, l'une restaurée et l'autre intacte, qui illustraient deux styles différents, se trouve sans doute à l'origine du récit de Pausanias. Le souci de faire la différence entre un style ancien sans doute archaïque et celui de son temps, ainsi que l'interdiction certainement symbolique de procéder à une autre restauration de ce type témoignent de la vision antiquaire de Pausanias et de son époque. Mais ils montrent aussi que les restaurations des statues anciennes dans le style contemporain étaient couramment pratiquées au $\mathrm{II}^{\mathrm{e}}$ siècle de notre ère.

\section{L'épiskève: les travaux de Télésinos et d'Aristandros à Délos}

Le mot épiskevè (émı état des sculptures dans un sens plus concret, privé de connotations symboliques. Sur ce point, Délos fournit à nouveau plusieurs attestations intéressantes ${ }^{46}$. Au début du III $^{\mathrm{e}}$ siècle av. J.-C., les Déliens honorent le sculpteur athénien Télésinos

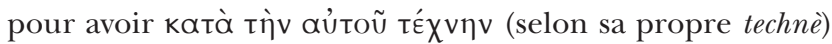

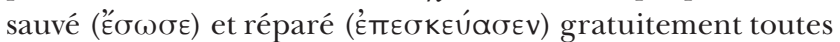
les statues du sanctuaire qui avaient besoin de réparation

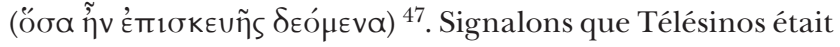
en premier lieu chargé par le peuple délien de réaliser une statue en bronze d'Asclépios et une autre en marbre de la reine Stratonikè. La renommée de Télésinos (ou Télésias) suffit à expliquer l'ampleur de son bienfait et les honneurs rendus par les Déliens. Son activité à Délos coïncide probablement avec la réalisation des statues colossales en marbre de Poséidon et d'Amphitrite sur l'île voisine de Ténos ${ }^{48}$.

L'épiskevè des sculptures est également attestée par les signatures des «sculpteurs-restaurateurs » gravées sur les 
socles des œuvres restaurées. Trois signatures d'Aristandros,

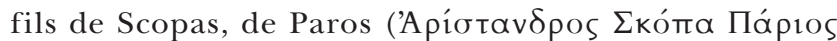

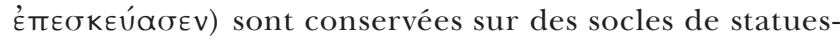
portraits en marbre d'officiers romains, réalisées pour l'Agora des Italiens à Délos par Agasias, fils de Ménophilos, d'Éphèse

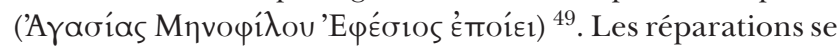
situent après le sac de l'île par les troupes de Mithridate (88 av. J.-C.) et concernent des œuvres créées seulement quelques années auparavant. La haute compétence dans le travail du marbre, liée à l'origine parienne et à la descendance familiale -Aristandros était l'arrière-petit-fils de Scopas, sculpteur célèbre du IV ${ }^{\mathrm{e}}$ siècle - était plus que suffisante aux Déliens pour lui confier la remise en état des œuvres créées par l'éminent Agasias d'Éphèse, lui aussi appartenant à une famille renommée de sculpteurs. Certaines sculptures déliennes conservent peut-être le souvenir des restaurations par Aristandros et son équipe. L'effigie-portrait d'Ofellius Ferus au Musée de Délos présente des réparations au niveau du pan de drapé qui tombe sur le dos et du pied gauche ${ }^{50}$. Conservé au Musée National, le Gaulois blessé de l'Agora des Italiens comporte aussi des raccords assez problématiques au haut du torse et de la tête ${ }^{51}$.

\section{La restauration du Zeus d'Olympie par Damophon de Messène}

Un autre sculpteur renommé a été engagé à la restauration du colosse chryséléphantin de Zeus à Olympie, chef-d'œuvre de Phidias. D’après Pausanias (IV, 31, 6), les Eléens ont honoré Damophon de Messène pour avoir rajusté avec la plus grande

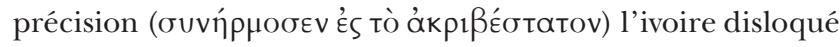

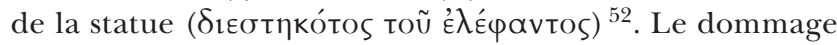
doit être localisé au niveau des joints fragiles des plaques d'ivoire qui revêtaient les parties nues de la figure, et le rajustement effectué à l'aide d'une colle et/ou de chevilles métalliques. Ce travail très délicat, exigeant une haute expertise technique, a été accompli par un sculpteur maître de différentes techniques, spécialisé dans les acrolithes et la réalisation de sculptures colossales par assemblage d'un grand nombre de pièces de marbre ${ }^{53}$. Le groupe cultuel de Déméter et Corè, autrefois érigé dans le temple de Lycosoura (Arcadie) et aujourd'hui conservé au Musée National, témoigne de l'expertise de Damophon (voir les dispositifs de raccord visibles sur les parties conservées du trône et des figures assises) ${ }^{54}$. La restauration de la statue de Zeus doit avoir eu lieu à la fin du $\mathrm{III}^{\mathrm{e}}$ ou au début du $\mathrm{II}^{\mathrm{e}}$ siècle av. J.-C., à savoir avant la création du groupe de Lycosoura daté des environs de 180 et dont le style classicisant semble avoir été adopté suite au contact avec l'œuvre de Phidias. Un tremblement de terre survenu en 193/2 et ayant causé des dommages au temple de Zeus peut avoir atteint la statue chryséléphantine qui se trouvait à l'intérieur et il est possible qu'à cette occasion, Damophon ait réparé aussi les sculptures tympanales ${ }^{55}$. Plusieurs phases de restauration datant du $\mathrm{IV}^{\mathrm{e}}$ au I $\mathrm{I}^{\mathrm{er}}$ siècle av. J.-C. ont été effectivement reconnues sur l'ensemble du décor sculptural du temple ${ }^{56}$. Or les éléments du groupe de Lycosoura comportent également une variété d'indices techniques plaidant en faveur d'une restauration à l'époque d'Hadrien, également suggérée par le contexte archéologique et historique ${ }^{57}$.

\section{Conclusion}

Même si la restauration des sculptures en Grèce ancienne ne répond pas à l'esthétique et à la déontologie de la restauration à l'époque contemporaine, même si les modifications apportées aux ouvres apparaissent incompatibles avec une vision d'œuvre d'art telle que nous la concevons aujourd'hui, la thérapéia pourvoyait à la nécessité concrète de conserver et, en cas d'altération, de rétablir la forme primitive et la lisibilité d'un objet en sorte qu'il continue à remplir la fonction précise à laquelle il était destiné. Le but ultime des opérations était de supprimer le plus possible toute trace des dégâts, de faire en sorte que l'objet parût intact, condition nécessaire pour qu'il soit digne de sa fonction cultuelle, votive, funéraire, honorifique. En plus des modalités de la genèse, approfondir la recherche sur l'histoire des œuvres après leur mise en place, celle-ci ayant parfois duré plusieurs siècles et connu de multiples vicissitudes, contribuera à mieux cerner le faciès de la restauration et ses liens avec les différentes valeurs attachées aux œuvres sculptées à travers l'Antiquité.

\section{Notes}

Liste des sigles et abréviations de collections

ID: Corpus des Inscriptions de Délos, Paris. $I G$ : Inscriptiones Graecae, Berlin.

1. Leka, 2003; 2008. La publication de l'étude étant en cours de préparation, mes remerciements les plus chaleureux sont adressés à Brigitte Bourgeois qui m'a si généreusement invitée à participer à ce volume, et qui a contribué avec des conseils précieux à la rédaction de l'article. J.-C.). 2. IG VII, 4149, 1. 17-21 (vers 50 av.

3. Sur le sanctuaire du Ptoion et sur sa statuaire, Ducat, 1971.
4. Voir article de B. Bourgeois dans le présent volume.

5. Vitruve, De l'architecture, VII, 9, 3-4; Pline, Histoire naturelle, XXXIII, 122; Plutarque, Euvres Morales, 287b-c et d.

6. Plutarque, Euvres Morales, 74d.

7. Sur ce texte, Papachatzis, 1979, p. 268, 484; Mansfield, 1985, p. 389 (note $35), 562, n^{\circ} 5$ (a) ; Lapatin, 2001, p. 86, 177; Muller-Dufeu, 2002, p. 322-323, n 919.

8. Voir Hesych., s.v. paıઈpuviǹs, «l'homme qui entretient $(\theta \varepsilon \rho \alpha \pi \varepsilon u ́ \omega v)$ la

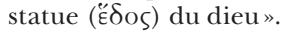

9. Donnay, 1967; Mansfield, 1985, p. 559-563; Lapatin, 2001, p. 153; Linant de Bellefonds, 2004, p. 423, n 34-37.
10. Sur le traitement des statues à l'huile d'olive et aux huiles parfumées, Leka, 2012, p. 279-284, 289.

11. Sur la technique de fabrication des colosses chryséléphantins, Lapatin, 2001, p. 68-78 (Athéna du Parthénon) et 81-83 (Zeus d'Olympie).

12. Pausanias, V, 11, 10-11; Papachatzis 1979, p. 261, 480; Lapatin, 2001, p. 62 , 85-86, 176-177; Muller-Dufeu, 2002, p. 310$311, n^{\circ} 872$

13. Sur ces réservoirs, Lapatin, 2001, p. 79-80 (temple d'Olympie), p. 85-86 (Parthénon), fig. 175.

14. Remplis d'eau et d'huile, ces réservoirs avaient aussi des qualités esthétiques. Du fait de son taux peu élevé d'évaporation et de sa capacité de réfléchir 
la lumière mieux que l'eau, l'huile était aussi destinée à illuminer l'intérieur du temple d'Olympie, dépourvu de fenêtres: Lapatin, 2001, p. 85-86, note 239 .

15. Lambrinoudakis, 2002.

16. Voir aussi Epiphanios, Panarion, II, 444: «l'huile d'olive versée autour des pieds devant la statue la conserve immortelle autant que possible »; Lapatin, 2001, p. 165, 171.

17. Sur la kosmésis annuelle des statues à Délos, Leka, 2012, 284-290.

18. Sur les passages relatifs à la kosmésis de l'Artémision et de l'Héraion, Homolle, 1890, p. 497-500; Tréheux, 1959, p. 440, note 1; Marcadé, 1969, p. 98-99; Bruneau, 1970 , p. 249-250; Mansfield, 1985, p. 534543, n ${ }^{\circ}$ 5-6; Linant de Bellefonds, 2004, p. 423, n $^{\circ} 26$; Prost, 2008, p. 101-103.

19. Mansfield, 1985 , p. $452-453\left(\mathrm{n}^{\circ} 1\right)$, 532-533.

20. Textes épigraphiques dans

Mansfield, 1985 , p. 543, n ${ }^{\circ} 7$ (Athènes), 544546, n 9 (Éphèse), 547, n 12 (Delphes)

21. Sur l'agalma de Dionysos, Vallois,

1922; Marcadé, 1969, p. 99; Bruneau, 1970 p. 312-317; Romano, 1982, p. 190-192; Mansfield, 1985 , p. 550-552, n ${ }^{\circ} 16$; Linant de Bellefonds, 2004, p. 423, n 27.

22. Sur le temple et la statue d'Aphrodite dédiée par Stésiléos à la fin du IV $^{\mathrm{e}}$ siècle, Vallois, 1944, p. 100, note 4 ; Tréheux, 1959, p. 426-444; Marcadé, 1969, p. 99-102, 225; Bruneau, 1970, p. 331-337; Yfantidis, 1984, p. 136; Mansfield, 1985 , p. 513-514 ( $\left.{ }^{\circ} 6\right), 552-553\left(\mathrm{n}^{\circ} 18\right), 565-566$ $\left(\mathrm{n}^{\circ}\right.$ 2) ; Manzelli, 1994, p. 108; Prost, 1999, p. 45 ; Linant de Bellefonds, 2004, p. 423 , $\mathrm{n}^{\circ} 24$.

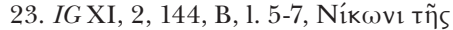

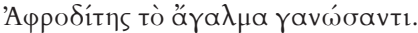

24. ID 338, Aa, 1. 42-43 et ID 354, 1. 77-78. Dans le premier cas, la rémunération du doreur s'élève à une drachme, y compris l'achat des feuilles d'or; dans le deuxième, elle atteint les deux drachmes, sans compter le prix des feuilles d'or (6 dr. et 4 oboles).

25. Par ex. ID 1417, A, II, 1. 1-3.

26. Quoique découvertes dans des secteurs très éloignés du sanctuaire, les deux dédicaces proviennent probablement de l'Aphrodision de Stésiléos: Tréheux, 1959, p. 449-450, note 7 .

27. Pausanias, IX, 40, 3-4; Marcadé, 1969, p. 101, 226; Papachatzis, 1981, p. 259, 510.

28. V. Chankowski dans ce volume.

29. IG XIV, 1277, signature d'Ôphélion sur une cuirasse du Louvre; Muller-Dufeu, 2002, n 2238.

30. Artiste actif à Rhodes autour de 340-330 av. J.-C. Il était à l'origine d'une lignée de sculpteurs que l'on arrive à suivre jusqu'à l'époque romaine: Muller-Dufeu, 2002, p. 763-765

31. Anthologie grecque, VI, 315-316; Muller-Dufeu, 2002, n 3048, 3049.

32. Sur la statue d'Artémis-Hécate «sur l'Île », Marcadé, 1969, p. 123-124; Bruneau, 1970 , p. 186-187; Mansfield, 1985, p. 513 $\left(n^{\circ} 5\right), 552\left(n^{\circ} 17\right)$; Linant de Bellefonds, 2004, p. 423, n $^{\circ} 28$ J.-C.)
33. $I G$ XI, 2, 287, A, 1. 114-115 (250 av.
34. ID 290, 1. 148-149 (246 av. J.-C.).

35. ID 290, 1. 151-153.

36. ID 290, 1. 228-243; Marcadé, 1969 , p. 100, 102; Mansfield, 1985, p. 514$517, \mathrm{n}^{\circ} 7$

37. La restitution est de B. Haussoulier

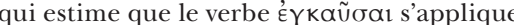
aux trois statues toutes entières, tandis que $\xi \tilde{u} \sigma \alpha \iota$ ne vaut que pour certaines parties qui avaient besoin de réparation. Voir le commentaire de F. Durrbach, dans ID 290. 38. Sur l'interprétation des mots anaplasis et othonia, Vallois 1944, p. 434. 39. Une mine ( $\mu v \tilde{\alpha})$ valait entre 400 et $600 \mathrm{gr}$

40. Les restitutions sont de B.

Haussoulier. Voir le commentaire de F. Durrbach, dans ID 290

41. IG XI, 2, 287, A, 1. 59-60; Marcadé 1969 , p. 99 ; Mansfield, 1985 , p. 553-554, $\mathrm{n}^{\circ} 19$.

42. IG XI, 2, 287, A, 1. 44, 100, 110, 122 ID $290,1.176$

43. Sur l'épikrasis, le culte et le sanctuaire des Dioscures à Délos, Tréheux, 1959 , p. 211-229; Bruneau, 1970, p. 379-399 Bruneau, Ducat, 1983, p. 258-260, n 123.

44. Sur le couros archaïque, la base et les fragments de la statue hellénistique (Musée de Délos, inv. A 3848, 4002-4005), Robert, 1952, p. 31-34, fig. 25-28.

45. Sur ce texte, Mansfield, 1985 , p. $549, \mathrm{n}^{\circ} 14$; Papachatzis, 1989, p. 364, 527.

46. V. Chankowski et M. Szewczyk dans ce volume.

47. IG XI 514 (300-294 av. J.-C.)

Marcadé, 1969, p. 100; Jockey, 1993, p. 346; Muller-Dufeu, 2002, n 2478; Linant de Bellefonds, 2004, p. 423, n 25

48. Sur l'activité artistique de Télésias Télésinos d'Athènes, Muller-Dufeu, 2002, $\mathrm{n}^{\circ} 1636,2478-2482$.

49. ID 1710; ID 1697; ID $1679+2494$ Marcadé, 1957, s.v. Aristandros; Jockey, 1993, p. 347; Muller-Dufeu, 2002, n 2577 2579 .

50. Barthe, Besnainou, 1988; Queyrel, 1991

51. Marcadé, 1969, p. 111-112; Jockey, 1993, p. 354; Marcadé, Queyrel, 2003, p. 5-30.

52. Sur ce passage de Pausanias, Papachatzis, 1979, p. 121 (note 1), 459 Stewart, 1990, p. 304; Lapatin, 2001, p. 78 (note 164), 83, 86 (note 251), 175; MullerDufeu, 2002, p. 324-325, n 920.

53. Sur Damophon de Messène, son œuvre et ses dates, Pollitt, 1986, p 312, note 8.2; Stewart, 1990, p. 303-304; Themelis, 1993a; 1993b; 1994; 1996; Muller-Dufeu, 2002, p. 814-823, n 2427-2436.

54. Sur le groupe de Lycosoura, Pausanias, VIII, 37, 3-5; Papachatzis, 1980, p. 336-338, 497-498; Stewart, 1990, p. 304, T 156; Muller-Dufeu, 2002, p. 818-819 $\mathrm{n}^{\circ} 2434$ 49

55. Themelis, 1996 , p. 172,182 note

56. Sur les restaurations du temple et des sculptures architecturales d'Olympie, Stucchi, 1952-1954; Ashmole, Yalouris, Frantz, 1967, p. 21-22, 179; Harrison, 1990, p. 170 , note 13 ; Younger, Rehak, 1996 ;

Symeonoglou, 1988; 2001; Trianti, 2002.
57. Sur la restauration du groupe de Lycosoura, Lévy, 1967; Donnay, 1967; Lévy, Marcadé, 1972; Harrison, 1990, p. 170, 172, notes 14-20; Stewart, 1990, p. 94-96.

\section{Bibliographie}

Ashmole B., Yalouris N., Frantz A., 1967, Olympia. The Sculptures of the Temple of Zeus, Londres.

Barthe G.-L., Besnainou D., 1988, "Restauration de l'effigie - portrait de Caius Offelius Ferus à Délos », BCH 112 , p. 414-432.

Bruneau Ph., 1970, Recherches sur les cultes de Délos à l'époque hellénistique et l'époque impériale, Paris.

Bruneau Ph., Ducat J., 1983, Guide de Délos, $3^{\mathrm{e}}$ éd., Paris, Athènes.

Donnay G., 1967, «Damophon de Messène et les Phaidyntai d'Olympie ", $B C H 91$, p. 546-551.

Ducat J., 1971, Les kouroi du Ptoion. Le sanctuaire d'Apollon Ptoieus à l'époque archaïque, Paris.

Harrison E., 1990, "Repair, Reuse and Reworking of Ancient Greek Sculpture", dans M. True, J. Podany (dir.), Marble. Art Historical and Scientific Perspectives on Ancient Sculpture, Malibu, The J. Paul Getty Museum, p. 163-184.

Homolle Th., 1890, «Comptes et inventaires des temples déliens de l'année 279 », BCH 14, p. 389-511.

Lambrinoudakis V., 2002, "Conservation and Research. New Evidence on a longliving Cult. The Sanctuary of Apollo Maleatas and Asklepios at Epidauros", dans M. Stamatopoulou, M. Yeroulanou (dir.), Excavating Classical Culture. Recent Archaeological Discoveries in Greece, Oxford, p. 213-224.

Lapatin K., 2001, Chryselephantine Statuary in the Ancient Mediterranean World, Oxford.

Leka E., 2003, «Restaurations antiques de sculptures archaïques de l'Acropole d'Athènes ", dans V. Gaggadis-Robin, Ph. Jockey (dir.), Approches techniques de la sculpture antique, Bulletin archéologique du CTHS 30, p. 17-31.

Leka E., 2012, «L'emploi des huiles, des onguents et des parfums dans l'entretien des statues en Grèce ancienne", dans D. Frère, L. Hugot (dir.), Les huiles parfumées en méditerranée occidentale et en Gaule, VIII siècle av.VIII ${ }^{e}$ siècle apr. J.-C., Rennes, p. 277-290

Lévy E., 1967, «Sondages à Lykosoura», $B C H$ 91, p. 518-545.

Lévy E., Marcadé J., 1972, «Au Musée de Lykosoura », BCH 96, p. 967-1004.

Linant de Bellefonds, P. (dir.) 2004, «Rites et activités relatifs aux images de culte », ThesCRA II (Los Angeles) p. $417-507$.

Mansfield J., 1985, The Robe of Athena and the Panathenaic Peplos, PhD Dissertation, University of California, Berkeley, Ann Arbor.

Manzelli V., 1994, La policromia nella statuaria greca arcaica, Rome. 
Marcadé J., 1957, Recueil des signatures de sculpteurs grecs, II. Délos, Athènes, Paris.

Marcadé J., 1969, Au Musée de Délos, Athènes, Paris.

Marcadé J., Queyrel F., 2003, «Le Gaulois blessé de Délos reconsidéré ", MonPiot 82 , p. 5-97.

Muller-Dufeu M., 2002, La sculpture grecque. Sources littéraires et épigraphiques, Paris.

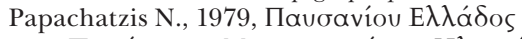

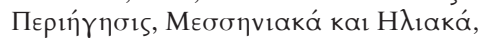
Athènes.

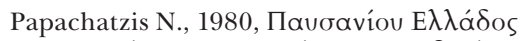

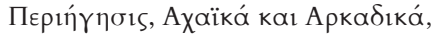
Athènes.

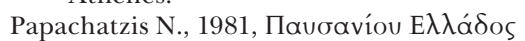

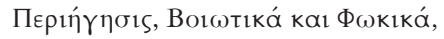
Athènes.

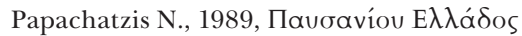

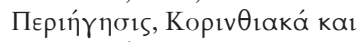
$\Lambda \alpha$ k $\omega v i k \alpha ́$, Athènes.

Pollitt J. J., 1986, Art in the Hellenistic Age, Cambridge.

Prost Fr., 1999, «La statue cultuelle d'Apollon à Délos », REG 112, p. 37-60.

Prost Fr., 2008, «L'odeur des dieux en Grèce ancienne. Encens, parfums et statues de culte», dans L. Bodiou, D. Frère, V. Mehl (dir.), Parfums et odeurs dans l'Antiquité, Rennes, p. 97-103.

Queyrel F., 1991, «C. Offelius Ferus », BCH 115 , p. 404-405.

Robert F., 1952, Trois sanctuaires sur le rivage occidental. Dioscourion, Asclépiéion, sanctuaire anonyme (Leucothion?), Paris.
Romano I. Bald, 1982, Early Greek Cult Images, PhD Dissertation, University of Pennsylvania, Ann Arbor.

Stewart A., 1990, Greek Sculpture. An Exploration, New Haven, Londres.

Stucchi S., 1952-54, «La decorazione figurata del Tempio di Zeus ad Olimpia ", ASAtene 14-16, p. 75-129.

Symeonoglou S., 1988, « Mía kaıvoúpı

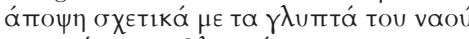

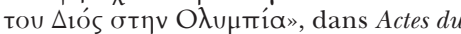
$X I I^{e}$ Congrès international d'archéologie classique, Athènes, p. 257-267.

Symeonoglou S., 2001, «Les maîtres d'Olympie» dans A. Pasquier (dir.), Olympie. Cycle de conférences, Musée du Louvre, 1999, Paris, p. 127-151.

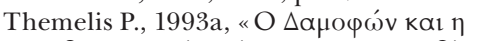

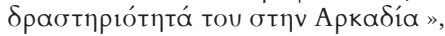
dans O. Palagia, W. Coulson (dir.), Sculpture from Arcadia and Laconia, Oxford, p. 99-109.

Themelis P., 1993b, «Damophon von Messene - sein Werk im Licht des neuen Ausgrabungen ", AntK36, p. 24-40.

Themelis P., 1994, "Damophon of Messene. New Evidence”, dans K. Sheedy (dir.), Archaeology in the Peloponnese, Oxford, p. 1-37.

Themelis P., 1996, "Damophon", dans J.J. Pollitt, O. Palagia (dir.), Personality in Greek Sculpture, Cambridge, p. 154-185.

Trianti I., 2002, «Neue technische Beobachtungen an den Skulpturen des
Zeustempels von Olympia », dans Olympia 1875-2000. 125 Jahre deutsche Ausgrabungen, Mayence, p. 281-300.

Vallois R., 1922, «L'agalma des Dionysies de Délos », BCH 46, p. 94-112.

Vallois R., 1944, L'architecture hellénique et hellénistique à Délos jusqu'à l'éviction des Déliens (166 av. J.-C.), I. Les Monuments, Paris.

Yfantidis K., 1984, Die Polychromie der hellenistischen Plastik, Mayence.

Younger J. G., Rehak P., 1996, “Technical Observations on the Sculptures of the Temple of Zeus at Olympia”, AJA 100, p. 367-368.

\section{Documents inédits}

Jockey Ph., 1993, Techniques et ateliers de sculpture à Délos à l'époque hellénistique, thèse de doctorat, Université Paris XNanterre.

Leka E., 2008, L'entretien et la restauration des sculptures en Grèce ancienne: le cas de la sculpture archaïque, thèse de doctorat, Université de Paris I (PanthéonSorbonne).

Tréheux J., 1959, Études critiques sur les inventaires de l'Indépendance Délienne, thèse de doctorat, Paris. 